\title{
Evaluation for Antioxidative Properties of Phlorotannins Isolated from the Brown Alga Eisenia bicyclis, by the H-ORAC Method
}

\author{
Yohei Fujii $^{1,2}$, Reiji Tanaka ${ }^{1,2}$, Hideo Miyake ${ }^{1,2}$, Yutaka Tamaru ${ }^{1}$, Mitsuyoshi Ueda ${ }^{2,3}$, \\ Toshiyuki Shibata ${ }^{1,2^{*}}$ \\ ${ }^{1}$ Major of Life Sciences, Graduate School of Bioresources, Mie University, Mie, Japan; ${ }^{2}$ Japan Science and Technology Agency, \\ CREST, Tokyo, Japan; ${ }^{3}$ Division of Applied Life Sciences, Graduate School of Agriculture, Kyoto University, Kyoto, Japan. \\ Email: ${ }^{*}$ shibata@bio.mie-u.ac.jp
}

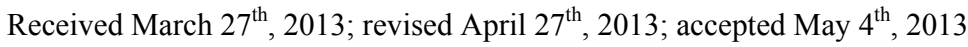

Copyright (C) 2013 Yohei Fujii et al. This is an open access article distributed under the Creative Commons Attribution License, which permits unrestricted use, distribution, and reproduction in any medium, provided the original work is properly cited.

\begin{abstract}
The antioxidative properties of phlorotannins isolated from the brown alga Eisenia bicyclis were measured using the H-ORAC (Hydrophilic Oxygen Radical Absorbance Capacity) method. The ORAC values of phloroglucinol and it's oligomers: eckol, fucofuroeckol A, phlorofucofuroeckol A, dieckol, 8,8'-bieckol, were $2.57 \pm 0.14,4.97 \pm 0.36,9.82 \pm$ $0.70,8.97 \pm 0.89,10.22 \pm 0.85,8.62 \pm 0.92 \mu \mathrm{mol}$ Trolox equivalent/ $\mathrm{mol}$, respectively. With the exception of eckol, the ORAC values of tested phlorotannins were higher than those of the well-known antioxidants (epigallocatechin gallate, resveratrol and L-ascorbic acid) used as positive controls. As a result of comparing with known ORAC values, it was found that the dieckol and fucofuroeckol A had stronger antioxidant activity than representative polyphenols (e.g., kaempferol, quercetin, myricetin and chlorogenic acid) derived from terrestrial plants.
\end{abstract}

Keywords: Antioxidants; Brown Alga; Dieckol; Eisenia bicyclis; Fucofuroeckol A; H-ORAC Assay; Phlorotannins

\section{Introduction}

The brown alga Eisenia bicyclis, belongs to the order Laminariales within the class of Phaeophyceae [1]. The brown alga is distributed along the temperate coasts from the central to southern parts of Japan, and form a kelp bed called a "marine forest" in the sub-tidal zone [1]. In Japan, this brown alga has been used since ancient times as an industrial source of alginic acid, and is currently attracting attention as a new raw material in the field of biorefinery.

It is known that the E. bicyclis produces polyphenols called phlorotannins $[2,3]$. Most phlorotannins, with the exception of some compounds, are oligomers of phloroglucinol (1,3,5-trihydroxybenzene), and can be divided into six categories, fucols, phlorethols, fucophlorethols, fuhalols, isofuhalols, and eckols [2,4]. Previously, we isolated the phlorotannins; eckol (phloroglucinol trimer), phlorofucofuroeckol A (a pentamer), dieckol, and 8,8'bieckol (hexamers) from E. bicyclis, and reported their distribution [3], 1,1-diphenyl-2-picrylhydrazyl (DPPH)-

${ }^{*}$ Corresponding author. radical and superoxide anion radical scavenging activities [5].

Recently, the ORAC assay has received much attention as a new in vitro method for measuring antioxidant activity [6], and is used to evaluate the antioxidative potencies of vegetables, fruits, their processed products, and phytochemicals [6-10]. In the case of phlorotannins, Parys et al. [11] isolated fucophlorethols from the brown alga Fucus vesiculosus, and reported their H-ORAC values. The ORAC values of eckols, however, are still obscure.

In this study, we isolated phloroglucinol and eckols from the brown alga E. bicyclis, and evaluated their antioxidative properties using the H-ORAC method. From comparison with well-known antioxidants, the availability of phlorotannins as antioxidants was also discussed.

\section{Materials and Methods}

\subsection{Materials}

The brown alga Eisenia bicyclis was collected from the coasts of the Itoshima Peninsula $\left(33^{\circ} 37^{\prime} \mathrm{N}, 130^{\circ} 10^{\prime} \mathrm{E}\right)$ in 
Fukuoka prefecture, Japan. The alga was washed with filtered seawater, air-dried and pulverized. The algal powder was stored at $-30^{\circ} \mathrm{C}$ until used.

Epigallocatechin gallate (EGCG) was kindly donated by Kurita Water Industries (Tokyo, Japan). Resveratrol and L-ascorbic acid were obtained from Wako Pure Chemical Industries (Osaka, Japan).

All reagents used in this study were of analytical grade.

\subsection{Extraction and Purification of Phlorotannins}

The extraction of the phlorotannins from the algal powder was carried out using the same method described in previous reports $[5,12]$. Each of the phlorotannins in the crude extracts was partially purified on a silicic acid column according to the same method described in a previous report [5]. Further purification of the phlorotannins was carried out using a preparative HPLC system. The HPLC system consisted of LC-6AD pumps (Shimadzu, Kyoto, Japan), a CBM-20A system controller (Shimadzu, Japan), a SPD-20A UV detector (Shimadzu, Kyoto, Japan), and an Inertsil ODS-3 column (10 mm I.D. $\times 250 \mathrm{~mm}$, GL Science, Tokyo, Japan). Elution was performed at a flow-rate of $4.7 \mathrm{ml} / \mathrm{min}$ with a linear gradient from $30 \% \mathrm{MeOH}$ to $100 \% \mathrm{MeOH}$ for $20 \mathrm{~min}$, and maintained for $20 \mathrm{~min}$. The UV detector was set at 290 $\mathrm{nm}$. The purity was confirmed by three-dimensional HPLC, using a photodiode array detector (SPD-M10AV, Shimadzu, Kyoto, Japan) with an Inertsil ODS-3 column (4.6 mm I.D. $\times 250 \mathrm{~mm}$, GL Science, Tokyo, Japan) $[5,12]$. The identification of the purified phlorotannins was carried out using a LC/MS system (6120 Quadrupole LC/MS with 1260 Series HPLC System, Agilent, CA, USA).

\subsection{H-ORAC Assay}

The H-ORAC assay of phlorotannins was performed according to the method of Watanabe et al. [6] with slight modification. Terrestrial polyphenols (EGCG and resveratrol) and an antioxidant vitamin (L-ascorbic acid) were used as positive controls [9,10]. 6-Hydroxy-2,5,7,8-tetramethylchroman-2-carboxylic acid (Trolox) standards $(3.125,6.25,12.5,25 \mu \mathrm{M})$, fluorescein $(110 \mathrm{nM})$, and 2,2'-azobis(2-amidinopropane) dihydrochloride (AAPH) $(31.7 \mathrm{mM})$ solutions were prepared separately by dissolving in a $75 \mathrm{mM}$ phosphate buffer $(\mathrm{pH}$ 7.4). Each sample was dissolved in $\mathrm{MeOH}(100 \mu \mathrm{g} / \mathrm{ml})$. The phosphate buffer was also used for dilution of the sample solutions.

The measurement was carried out using a 96-well black plate (Costar 3915, Corning, NY, USA) with plate seal (Q-Stick adhesive sheets for qPCR, 4 titude, Surrey,
UK) and a 96-well microplate reader (Infinite 200, Tecan Japan, Kanagawa, Japan). The sample solution $(10 \mu \mathrm{l})$, fluorescein $(115 \mu \mathrm{l})$ and the phosphate buffer $(25 \mu \mathrm{l})$ were added to the sample wells in the plate. Fluorescein $(115 \mu \mathrm{l})$, Trolox, or the phosphate buffer (blank) $(35 \mu \mathrm{l})$, were added to the Trolox wells or blank wells, respecttively. Each plate was incubated at $37^{\circ} \mathrm{C}$. To prevent error of the ORAC value, the plate was preincubated at $37^{\circ} \mathrm{C}$ for $5 \mathrm{~min}$ before each measurement. The phosphate buffer used for the AAPH solution was also incubated at $37^{\circ} \mathrm{C}$ for $30 \mathrm{~min}$ before each measurement, and then the AAPH solution was prepared $[7,8]$. The fluorescence intensity (excitation at $485 \mathrm{~nm}$, emission at $528 \mathrm{~nm}$ ) was monitored every $2 \mathrm{~min}$ for $90 \mathrm{~min}$ by a microplate reader. The assays were made in three independent measurements. The area under the curve (AUC) of the fluorescence decay from 8 to 90 min after the addition of AAPH solution was calculated for each well. The net AUC was calculated by subtracting AUC for the sample, or Trolox standard, from that for the blank. A calibration curve was constructed from the net AUCs of Trolox standard solutions, and a power approach was fitted to the results. The $\mathrm{H}-\mathrm{ORAC}$ value for each sample was calculated on the basis of the standard curve for Trolox and expressed as $\mu \mathrm{mol}$ of Trolox equivalents (TE) $/ \mu \mathrm{mol}$ of samples. The final ORAC values were expressed as the mean $\pm \mathrm{SD}$ ( $\mathrm{n}$ $=3)$.

\section{Results and Discussion}

Figure 1 shows the chemical structures of each of the phloroglucinol and eckols isolated from the brown alga $E$. bicyclis. These phlorotannins were refined to a purity of $98 \%$ or more by column chromatography and preparative HPLC (data not shown), and used in an H-ORAC assay.

The ORAC measures the scavenging activity against peroxyl radicals induced by AAPH at $37^{\circ} \mathrm{C}$, using fluorescein as a fluorescence probe [6-10]. The ORAC value is widely used as one of the quantitative standards that shows the antioxidative potency of an antioxidant [6-10]. Generally, antioxidants can be classified by their solubility into two groups: hydrophilic antioxidants (e.g., ascorbic acid and majority of polyphenols) and lipophilic antioxidants (e.g., tocopherols and carotenoids) [13]. The $\mathrm{H}$-ORAC assay is a method that can measure the titer of a hydrophilic antioxidant, and so was used to evaluate the antioxidative properties of phlorotannins in this study. The most suitable dilution ratio of the sample solution for the H-ORAC measurement was selected according to the method of Watanabe et al. [6]. As a result, the AUC of 25X and 30X diluted solutions of each sample solution $(100 \mu \mathrm{g} / \mathrm{ml})$ were located between $3.125 \mu \mathrm{M}$ Trolox AUC, and $25 \mu \mathrm{M}$ Trolox AUC respectively (data not shown). Therefore, sample solutions diluted $25 \mathrm{X}$ and 


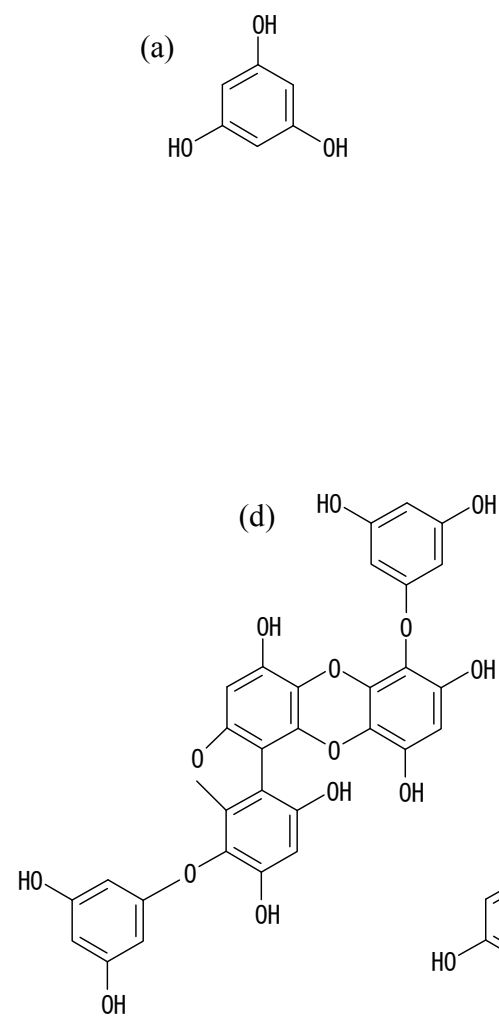<smiles>Oc1cc(O)cc(Oc2c(O)cc(O)c3c2Oc2c(O)cc(O)cc2O3)c1</smiles>

(e)<smiles>Oc1cc(O)cc(I)c1</smiles><smiles>Oc1cc(O)cc(Oc2c(O)cc(O)c3c2Oc2c(O)cc4oc5cc(O)cc(O)c5c4c2O3)c1</smiles>

Figure 1. Chemical structures of phloroglucinol and phlorotannins isolated from the brown alga $E$. bicyclis. These compounds were isolated from crude phlorotannins of $E$. bycyclis by column chromatography and preparative HPLC. (a) phloroglucinol, (b) eckol, (c) fucofuroeckol A, (d) phlorofucofuroeckol A, (e) dieckol, (f) 8,8'-bieckol.

$30 \mathrm{X}$ were used for the experiment. Figure 2 shows the fluorescence decay curves in the presence of the sample solutions diluted $25 \mathrm{X}$ and $\mathrm{AAPH}$, as typical data. The calculated H-ORAC values of samples are shown in Table 1. Among phloroglucinol and it's oligomers, it was found that the compounds more than phloroglucinol tetramer had potent antioxidant activity with H-ORAC values in the range of $8.62-10.22 \mu \mathrm{M} \mathrm{TE} / \mu \mathrm{M}$. In contrast, the H-ORAC values of positive controls were in the range of $0.76-7.18 \mu \mathrm{M} \mathrm{TE} / \mu \mathrm{M}$. The data of EGCG and L-ascorbic acid were also almost in agreement with the values showed by Ishimoto et al. [10]. Although phloroglucinol and eckol had little antioxidant activities (HORAC values: $2.57 \mu \mathrm{M}$ and $4.97 \mu \mathrm{M}$ ) in the tested samples, they were more effective than L-ascorbic acid. Ishimoto et al. [10] reported the H-ORAC values of 43 terrestrial polyphenols and their metabolites. Compared with their H-ORAC values in the report, phlorotannins, in particular dieckol and fucofuroeckol A, had H-ORAC values that were higher than terrestrial polyphenols (ellagitannins, condensed tannins, chlorogenic acids, catechins and flavonoids). Therefore, it was suggested that phlorotannins had more potent antioxidant activity than a lot of the representative polyphenols (e.g., EGCG, resveratrol, kaempferol, quercetin, myricetin and chlorogenic acid).

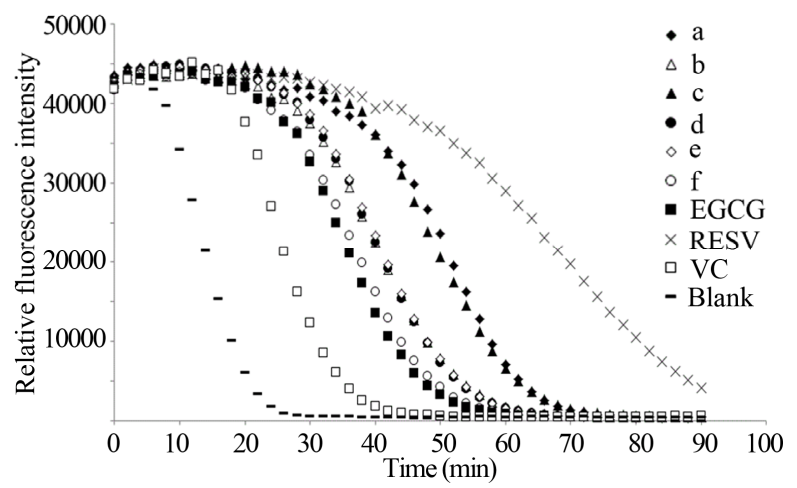

Figure 2. Fluorescence decay curves induced by AAPH in the presence of phlorotannins and positive controls. The figure shows the data of a $25 \times$ diluted solution of each sample solution $(100 \mu \mathrm{g} / \mathrm{ml})$ as typical data. (a) phloroglucinol, (b) eckol, (c) fucofuroeckol A, (d) phlorofucofuroeckol A, (e) dieckol, (f) 8,8'-bieckol, EGCG; epigallocatechin gallate, RESV; resveratrol, VC; L-ascorbic acid.

Phlorotannins, which are shown in Figure 1, are also commonly found in the Laminariaceous brown algae, Eisenia arborea, Ecklonia cava and Ecklonia kurome $[3,14,15]$. Previously, we showed that eckols had significant radical scavenging activities against the superoxide anion (50\% effective concentration values: $6.5-8.4 \mu \mathrm{M})$ 
Table 1. H-ORAC values of phloroglucinol and phlorotannins isolated from the brown alga $E$. bicyclis.

\begin{tabular}{cc}
\hline Samples & ORAC $(\mu \mathrm{mol} \mathrm{TE} / \mu \mathrm{mol})$ \\
\hline Phloroglucinol & $2.57 \pm 0.14$ \\
Phlorotannins & \\
Eckol & $4.97 \pm 0.36$ \\
Fucofuroeckol A & $9.82 \pm 0.70$ \\
Phlorofucofuroeckol A & $8.97 \pm 0.89$ \\
Dieckol & $10.22 \pm 0.85$ \\
8,8'-Bieckol & $8.62 \pm 0.92$ \\
Positive controls & \\
L-Ascorbic acid & $0.76 \pm 0.24$ \\
Epigallocatechin gallate & $4.65 \pm 0.80$ \\
Resveratrol & $7.18 \pm 0.24$ \\
\hline
\end{tabular}

The data is expressed as the mean \pm standard deviation from three independent measurements.

and DPPH (50\% effective concentration values: 12 - 26 $\mu \mathrm{M}$ ), and were more effective than L-ascorbic acid and $\alpha$-tocopherol [5]. Nagayama et al. [16] reported that phlorotannins had no toxicity following oral administration to mice. The evidence obtained in the previous and present studies suggest that phlorotannins are potent antioxidants and that Laminariaceae, which are rich in phlorotannins, are useful as a novel functional food or supplement with antioxidative properties.

\section{Acknowledgements}

This work was supported by the Japan Science and Technology Agency. We acknowledge that the English was revised by Mr. Shane LeGros (Eigo Step, Tsu, Japan).

\section{REFERENCES}

[1] T. Yoshida, "Marine Algae of Japan," Uchida Rokakuho Publishing, Tokyo, 1988.

[2] M. A. Ragan and K.-W. Glombitza, "Phlorotannins, Brown Algal Polyphenols," In: F. E. Round and D. J. Chapman, Eds., Progress in Phycological Research, Vol. 4, Biopress, Bristol, 1986, pp. 129-241.

[3] T. Shibata, S. Kawaguchi, Y. Hama, M. Inagaki, K. Yamaguchi and T. Nakamura, "Local and Chemical Distribution of Phlorotannins in Brown Algae," Journal of Applied Phycology, Vol. 16, No. 4, 2004, pp. 291-296. doi:10.1023/B:JAPH.0000047781.24993.0a

[4] C. D. Amsler and V. A. Fairhead, "Defensive and Sensory Chemical Ecology of Brown Algae," In: J. A. Callow, Ed., Advances in Botanical Research, Vol. 43, Elsevier, Amsterdam, 2006, pp. 1-91. doi:10.1016/S0065-2296(05)43001-3

[5] T. Shibata, K. Ishimaru, S. Kawaguchi, H. Yoshikawa and Y. Hama, "Antioxidant Activities of Phlorotannins Isola- ted from Japanese Laminariaceae," Journal of Applied Phycology, Vol. 20, No. 5, 2008, pp. 705-711. doi:10.1007/s10811-007-9254-8

[6] J. Watanabe, T. Oki, J. Takebayashi, K. Yamasaki, Y. Takano-Ishikawa, A. Hino and A. Yasui, "Method Validation by Interlaboratory Studies of Improved Hydrophilic Oxygen Radical Absorbance Capacity Methods for the Determination of Antioxidant Capacities of Antioxidant Solutions and Food Extracts," Analytical Sciences, Vol. 28, No. 2, 2012, pp. 159-165. doi:10.2116/analsci.28.159

[7] M. K. Ehlenfeldt and R. L. Prior, "Oxygen Radical Absorbance Capacity (ORAC) and Phenolic and Anthocyanin Concentrations in Fruit and Leaf Tissues of Highbush Brueberry," Journal of Agricultural and Food Chemistry, Vol. 49, No. 5, 2001, pp. 2222-2227.

doi:10.1021/jf0013656

[8] R. L. Prior, H. Hoang, L. Gu, X. Wu, M. Bacchiocca, L. Howard, M. Hampsch-Woodill, D. Huang, B. Ou and R. Jacob, "Assays for Hydrophilic and Lipophilic Antioxidant Capacity (Oxygen Radical Absorbance Capacity $\left(\mathrm{ORAC}_{\mathrm{FL}}\right)$ ) of Plasma and Other Biological and Food Samples," Journal of Agricultural and Food Chemistry, Vol. 51, No. 11, 2003, pp. 3273-3279. doi:10.1021/jf0262256

[9] C. Lucas-Abellán, M. T. Mercader-Ros, M. P. Zafrilla, M. I. Fortea, J. A. Gabaldón and E. Núñez-Delicado, "ORACFluorescein Assay to Determine the Oxygen Radical Absorbance Capacity of Resveratrol Complexed in Cyclodextrins," Journal of Agricultural and Food Chemistry, Vol. 56, No. 6, 2008, pp. 2254-2259. doi:10.1021/jf0731088

[10] H. Ishimoto, A. Tai, M. Yoshimura, Y. Amakura, T. Yoshida, T. Hatano and H. Ito, "Antioxidative Properties of Functional Polyphenols and Their Metabolites Assessed by an ORAC Assay," Bioscience, Biotechnology, and Biochemistry, Vol. 76, No. 2, 2012, pp. 395-399. doi:10.1271/bbb.110717

[11] S. Parys, S. Kehraus, A. Krick, K.-W. Glombitza, S. Carmeli, K. Klimo, C. Gerhäuser and G. M. König, "In Vitro Chemopreventive Potential of Fucophlorethols from the Brown Alga Fucus vesiculosus L. by Anti-Oxidant Activity and Inhibition of Selected Cytochrome P450 Enzymes," Phytochemistry, Vol. 71, No. 2-3, 2011, pp. 221229. doi:10.1016/j.phytochem.2009.10.020

[12] T. Shibata, Y. Hama, T. Miyasaki, M. Ito and T. Nakamura, "Extracellular Secretion of Phenolic Substances from Living Brown Algae," Journal of Applied Phycology, Vol. 18 , No. 6, 2006, pp. 787-794. doi:10.1007/s10811-006-9094-y

[13] B. Halliwell, "Antioxidants in Human Health and Disease," Annual Review of Nutrition, Vol. 16, 1996, pp. 3350. doi:10.1146/annurev.nu.16.070196.000341

[14] S.-H. Eom, Y.-M. Kim and S.-K. Kim, "Antimicrobial Effect of Phlorotannins from Marine Brown Algae," Food and Chemical Toxycology, Vol. 50, No. 9, 2012, pp. 3251-3255. doi:10.1016/j.fct.2012.06.028

[15] Y. Sugiura, K. Matsuda, Y. Yamada, M. Nishikawa, K. Shioya, H. Katsuzaki, K. Imai and H. Amano, "Isolation of a New Anti-Allergic Phlorotannin, Phlorofucofuroec- 
kol-B, from Edible Brown Alga, Eisenia arborea," Bioscience, Biotechnology, and Biochemistry, Vol. 70, No. 11, 2006, pp. 2807-2811. doi:10.1271/bbb.60417

[16] K. Nagayama, Y. Iwamura, T. Shibata, I. Hirayama and T.
Nakamura, "Bactericidal Activity of Phlorotannins from the Brown Alga Ecklonia kurome," Journal of Antimicrobial Chemotherapy, Vol. 50, No. 6, 2002, pp. 889-893. doi:10.1093/jac/dkf222 